\title{
Transverse myelitis following COVID-19 infection. What is the mechanism? A case report and literature review
}

\author{
Babak Alijani', Alia Saberi², Parham Niyasti ${ }^{3}$, Mohammad Haghani Dogahe ${ }^{4}$ \\ ${ }^{1}$ Road Trauma Research Center, Guilan University of Medical Sciences, Rasht, Iran \\ ${ }^{2}$ Neuroscience Research Center, Department of Neurology, Poursina Hospital, \\ Guilan University of Medical Sciences, Rasht, Iran \\ ${ }^{3}$ Department of Neurology, Guilan University of Medical Science, Rasht, Iran \\ ${ }^{4}$ Research Committee, School of Medicine, Guilan University of Medical Science, Rasht, Iran
}

\begin{abstract}
The severe acute respiratory syndrome coronavirus 2 (SARS-CoV-2) outbreak became one of the most global health challenges in the recent century. Transverse myelitis is a rare medical condition reported in few case reports following COVID-19 infection.

We present a 63 years old female with post-COVID-19 transverse myelitis. The patient had paraplegia combined with autonomic symptoms and T4 sensory level.

Initial treatment started with methylprednisolone. Since the patient did not respond to the prednisolone, plasmapheresis was considered for the patient. Unfortunately, the patient could not tolerate the plasmapheresis.

We considered rituximab for the next plan, but unfortunately, the patient died due to respiratory failure.

To date, there is no published study to evaluate different mechanisms of post/para COVID-19 transverse myelitis. However, the post/para COVID-19 transverse myelitis mechanism seems to be heterogeneous; the activation of a silent autoimmune disease can probably justify some unusual clinical presentations of a viral illness. Further studies are needed to categorize the underlying mechanisms of post/para COVID-19 transverse myelitis and effective treatments regarding the primary pathology.
\end{abstract}

Keywords: transverse myelitis, SARS-CoV-2, post/para COVID-19, autoimmune mechanism

\section{INTRODUCTION}

Severe acute respiratory syndrome coronavirus 2 (SARS-CoV-2) is one of the strains of human coronaviruses (HCoV) transmitted as RNA virus. SARS-CoV-2 infection can lead to several nervous system complications. Transverse myelitis (TM) referred to pathological infiltration of the whole cross-sectional area of the cord at one or more levels, leading to varying degrees of weakness, sensory alterations, and autonomic dysfunction (1).

Transverse myelitis may result from abnormal immune reaction or direct pathways, including angiotensin-converting enzyme-2 related pathway, trans-synaptic pathway, hematogenous and lym- phatic pathways, or migration of infected immune cells (2).

As far as we are aware, the ATM has bimodal peaks where between ages 10-19 and 30-39 years old, but in the setting of COVID-19 infection, the age distribution is much older (3).

However, the mechanisms mentioned above are suggested for post/para COVID-19 infectious TM; we believe the fundamental mechanism is heterogenous in each individual.

Herein the authors described a case of post-COVID-19 transverse myelitis and comprehensively reviewed the literature regarding COVID-19 associated transverse myelitis. 
We proposed a new probable mechanism that signifies an underlying silent immune disease in some patients may have a role in victims' response to SARS-COV-2 infection based on published case reports and certainly discuss recent experiences and management towards handling COVID-19 induced transverse myelitis.

\section{CASE PRESENTATION}

The patient was a 63 years old female referred to our hospital with paraplegia. The patient was hospitalized in COVID-19 referral hospital three weeks before by dry cough and short breathing, and positive throat COVID-19 PCR. The patient's respiratory symptoms recovered by hydroxychloroquine and azithromycin and supportive care. Two days after discharge, the patient developed progressive paraparesis, urinary incontinency, and back pain progressed rapidly, which led to the current admission.

Upon presentation, she was alert, and her $\mathrm{O}_{2}$ saturation was $98 \%$; she was vitally stable and afebrile. There were no associated cognitive and bulbar symptoms nor residual flue-like symptoms. The motor function was significantly disrupted. Bilateral upper limb forces were three out of five in strength (MRC), and lower limbs were utterly lost. Sensory level with hypoesthesia below the nipples. Pinprick, light touch, pain, temperature, and proprioception were diminished below the sensory level.

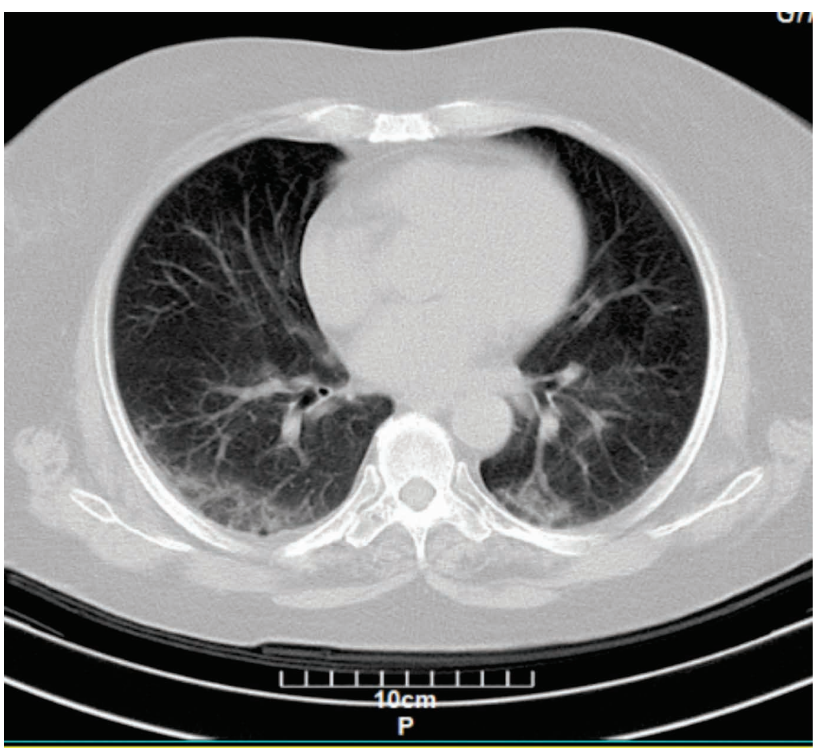

FIGURE 1. Thoracic CT imaging findings showing bilateral ground-glass opacity suggestive of COVID-19 infection
Left and right upper limbs deep tendon reflex (DTR) were normal, Hoffman was negative, lower limbs DTRs were lost, and plantar reflexes were mute.

A new chest CT scan was performed upon admission. findings were included bilateral and peripheral ground-glass opacities compatible with lung infection with COVID-19 virus (fig. 1).

MRI of the brain and entire spine was taken on the second day of admission. The significant signal change was seen in the T2 weighted sequence in the sagittal view from T5 to T8 (fig. 2), which involves mostly the gray matter in the axial view corresponding to longitudinally extensive transverse myelitis (fig. 3).

Initial lab data demonstrate very high D-Dimer, low procalcitonin, a left shift in neutropenia without leukocytosis, and a slight increase in ESR. A general systemic autoimmune study (rheumatoid factor, ANA, c-ANCA, p-ANCA, anti dsDNA, anti-CCP, anti-Ro, anti-La, $\mathrm{CH}_{50}, \mathrm{C}_{3}, \mathrm{C}_{4}, \mathrm{ACE}$ level, antiphospholipid antibody, anti-lupus cardiolipin, anti-AQP4 antibodies, anti-MOG antibodies) and syphilis serology, protein C and S levels, HIV and enterovirus testing performed for the patient. Metabolic and nutritional deficiencies were also considered for the patient, and the tests were not associated with the disease. All laboratory findings were normal. We tried for lumbar puncture, but it was not successful. Initial treatment with IV prednisolone ( $1 \mathrm{~g} /$ daily) started and continued for five days. The patient manifested hypocalcemia, anemia, and leukopenia. We decided on plasmapheresis regarding the progression of symptoms, but due to hemodynamic instability, the plasmapheresis was disrupted. Due to abnormal lab data and multiple organ involvement, the therapeutic team considered Rituximab to modify post-COVID-19 immunological processes.

At this time, the patient developed shortness of breathing. A new CT scan showed pleural effusion on the left side. An emergency surgical consult occurred for the evacuation of the effusion. At $8^{\text {th }} 8^{\text {th }}$ day of admission, the patient passed away in the setting of respiratory failure and multiple organ failure.

\section{DISCUSSION}

In this study, we presented a case of post-COVID-19 transverse myelitis 63-year-old female pre- 


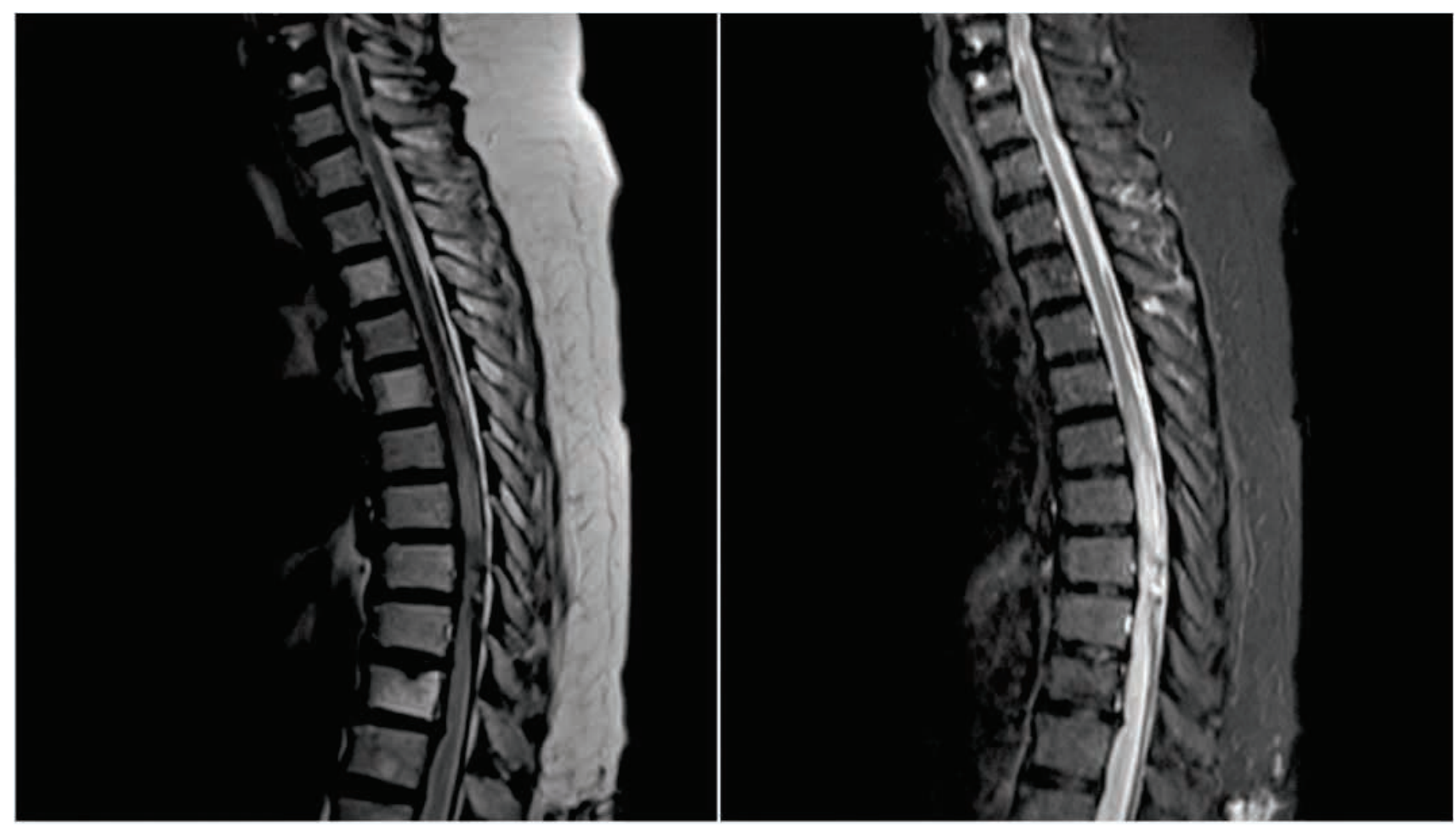

FIGURE 2. Sagittal T2-weighted scan shows longitudinal transverse myelitis extended from T5 to T8

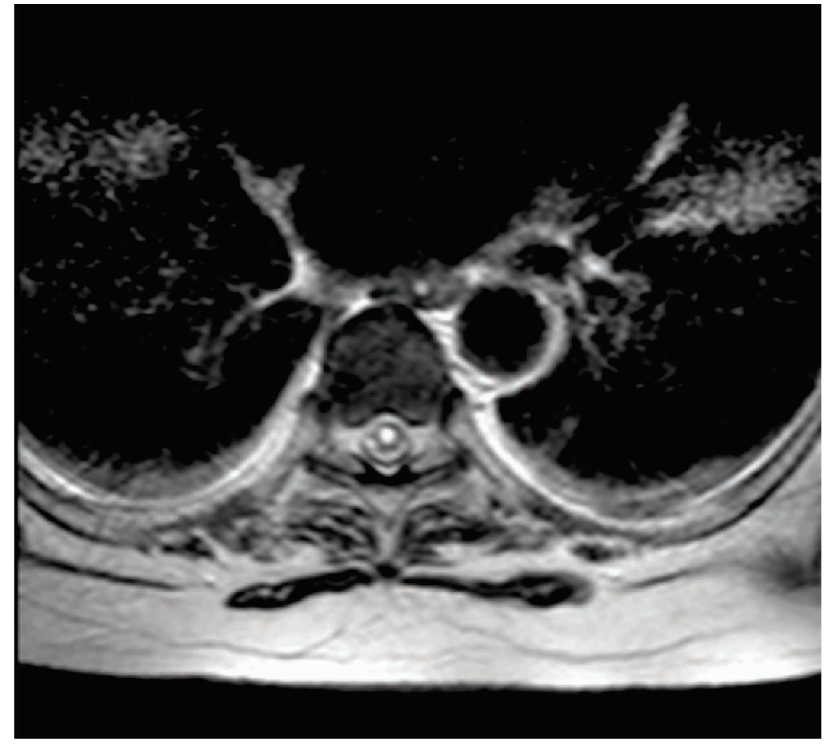

FIGURE 3. Axial T2 image of the dorsal spine showing central hyperintense signal suggestive for transverse myelitis

sented with rapidly progressive paraplegia continued to quadriplegia.

Table 1 shows published case reports of post/ para infectious COVID-19 TM to the best of our knowledge. TM may develop concomitant or a few days after SARS-CoV-2 infection.

Brain and whole spine MRI and the autoimmune panel are necessary to rule out several compressive or non-inflammatory myelopathies which can mimic idiopathic TM (4).
Emergencies such as cord compression or non-inflammatory myelopathies, which can mimic TM, must be ruled out by MRI of the entire spine (5). The typical finding of cord inflammation is hyperintensity in the T2 sequence which can appear in one or more cord segments $(6,7)$.

While systematic or local autoimmune diseases and spinal cord infarcts can result in acute transverse myelitis, in most cases, the etiology of TM remains unknown (idiopathic TM). Idiopathic TM appears to result from the post/para infectious autoimmune process $(8,9)$.

Herein we discuss sixteen para/post-COVID ATM published case report published in MEDLINE data base. the mean age of patients was $48.69 \pm 19.67$ (ranging from 3 to 70 years old), eight cases were female, and eight cases were male $(50 \%)$. Seven patients $(43.75 \%)$ did not have significant past medical history and comorbidity, but hypertension was more prevalent among patients with significant comorbidities (66.6\%). It has been estimated that the majority of idiopathic TM cases were below 39 years of age, and about $20 \%$ of patients are under the age of 18 years, but in current published case reports, only one case was below 18 years old. In contrast, in the setting of COVID-19 induced, most patients were higher than 50 years old $(3,10)$. One probable idea is that the virus affects children much less (11). It might 


\begin{tabular}{|c|c|c|c|c|c|c|c|c|c|c|c|c|}
\hline 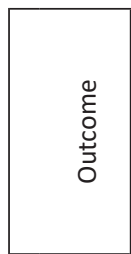 & 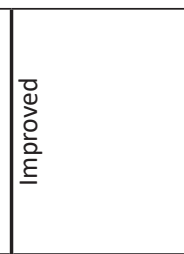 & 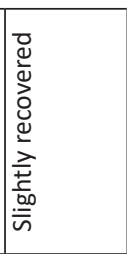 & 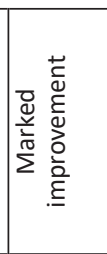 & 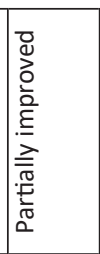 & 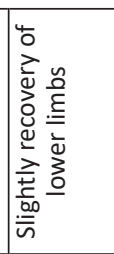 & 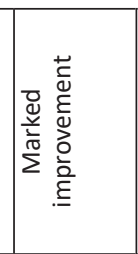 & 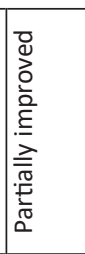 & 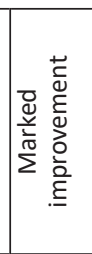 & 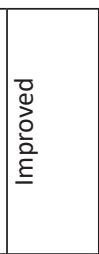 & 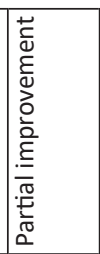 & 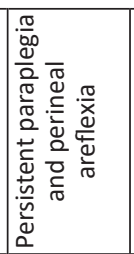 & 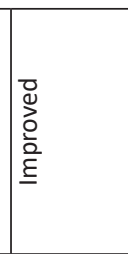 \\
\hline 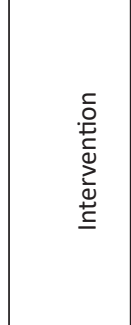 & 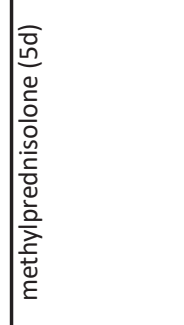 & 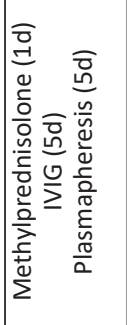 & 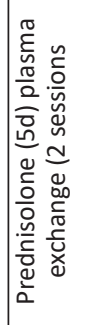 & 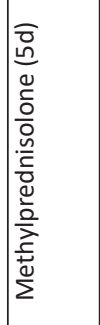 & 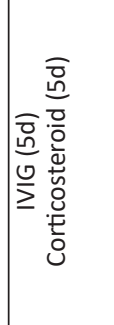 & 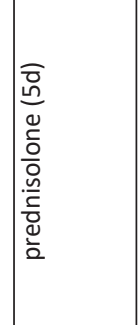 & 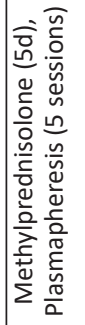 & 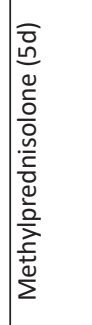 & 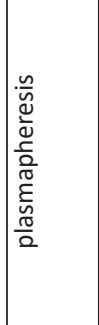 & 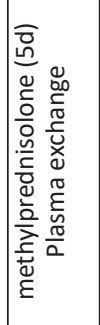 & 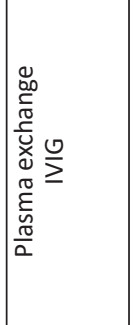 & 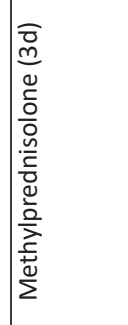 \\
\hline 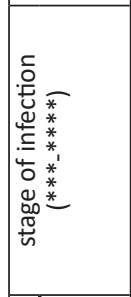 & 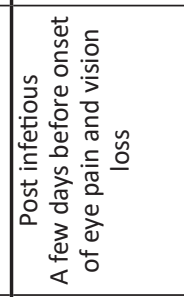 & 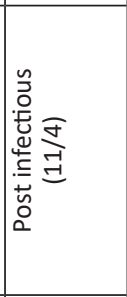 & 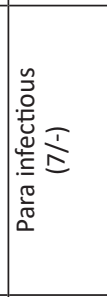 & 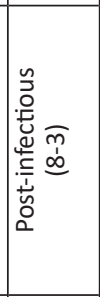 & 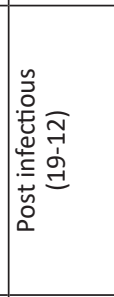 & 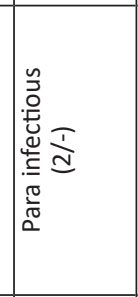 & 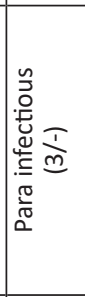 & 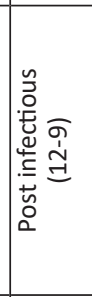 & 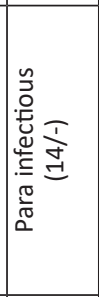 & 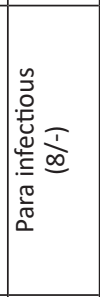 & 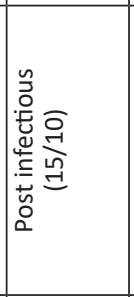 & 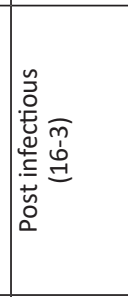 \\
\hline 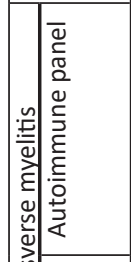 & 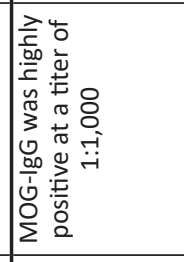 & 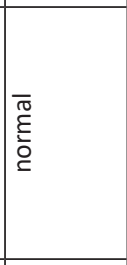 & 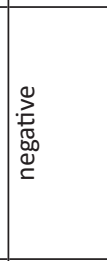 & 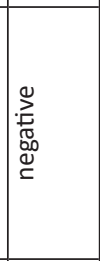 & 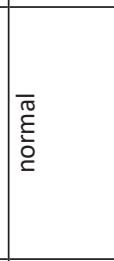 & 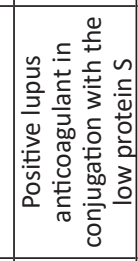 & 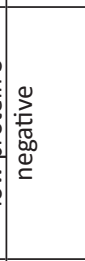 & $\begin{array}{l}\overline{\widetilde{J}} \\
\underline{\xi} \\
\check{\check{c}}\end{array}$ & 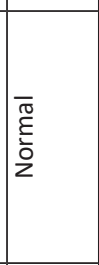 & 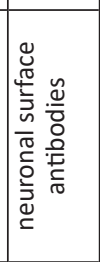 & 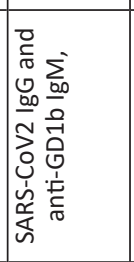 & 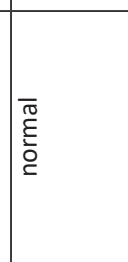 \\
\hline 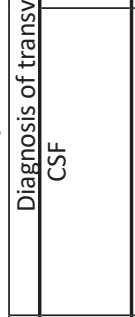 & 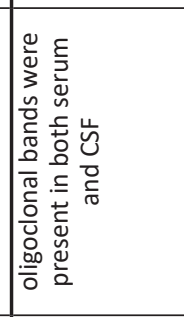 & 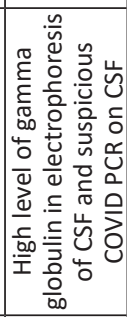 & 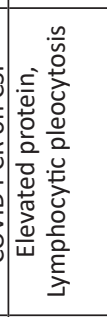 & 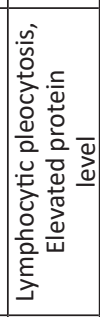 & 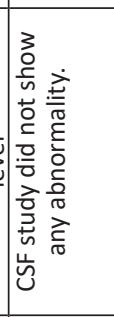 & 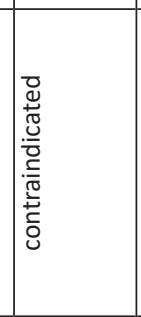 & 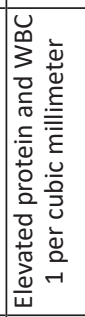 & 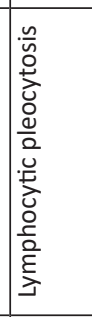 & 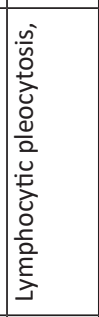 & 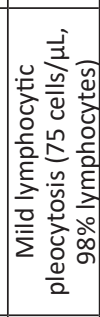 & 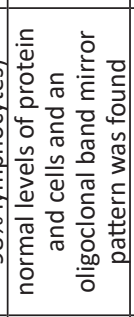 & 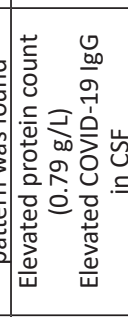 \\
\hline 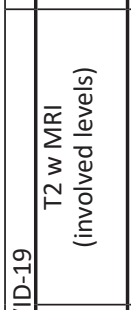 & 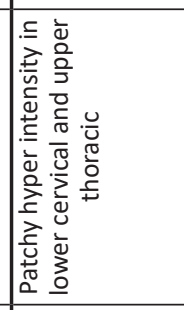 & 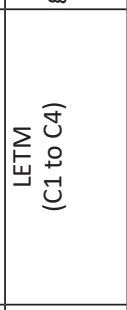 & 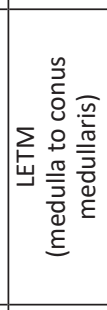 & 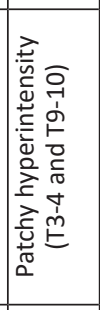 & 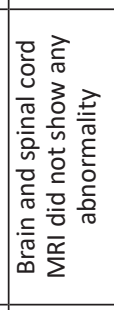 & 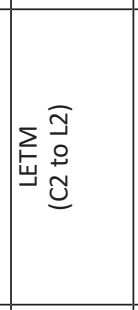 & $\sum_{\underline{\underline{W}}}$ & 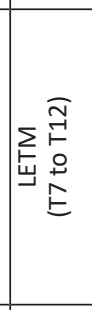 & 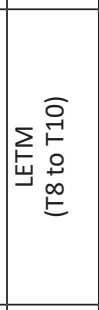 & 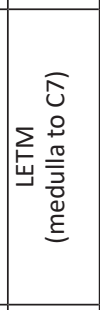 & $\sum_{i} \overline{\underline{i}}$ & 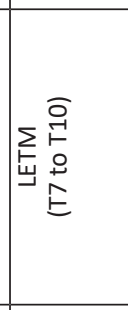 \\
\hline 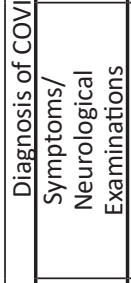 & 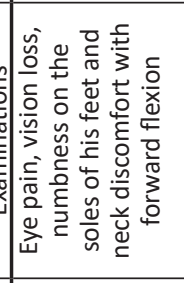 & 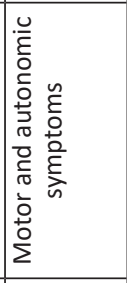 & 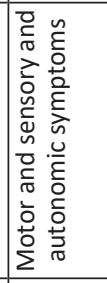 & 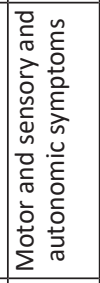 & 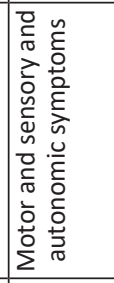 & 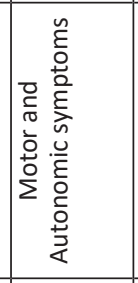 & 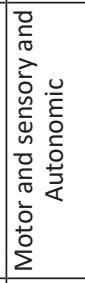 & 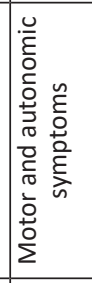 & 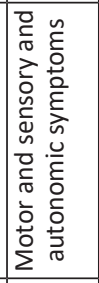 & 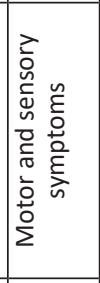 & 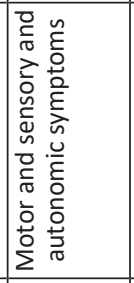 & 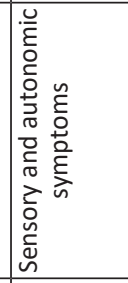 \\
\hline 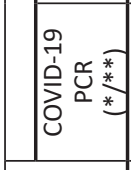 & 王 & $I$ & $\begin{array}{l}\text { I } \\
\text { 土 }\end{array}$ & $\frac{1}{ \pm}$ & : & $\mp$ & 玉 & $\frac{1}{\text { 主 }}$ & $\stackrel{1}{ \pm}$ & $\Phi$ & $I$ & $\mp$ \\
\hline 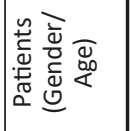 & $\stackrel{\frac{0}{\pi}}{\Sigma}$ & $\therefore \frac{0}{20}$ & 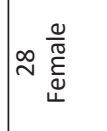 & $\therefore \frac{0}{20}$ & 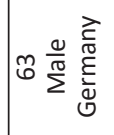 & $\approx \frac{0}{m} \frac{0}{\pi}$ & $\overrightarrow{0} \frac{\frac{0}{\tilde{T}}}{\tilde{E}}$ & $\stackrel{\sim}{\sim} \frac{0}{\pi}$ & in & ه & $ং$ & $8 \frac{0}{\frac{0}{\pi}}$ \\
\hline 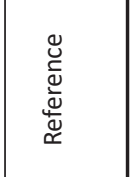 & 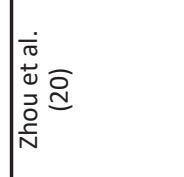 & 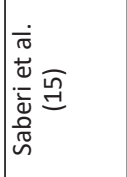 & 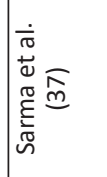 & 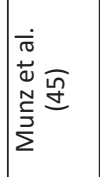 & 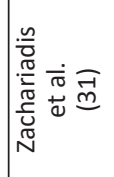 & 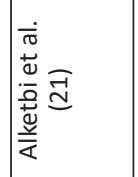 & 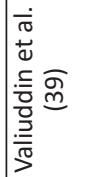 & 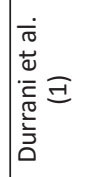 & 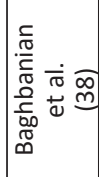 & 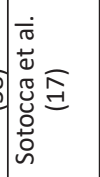 & 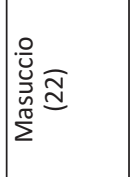 & 总 \\
\hline
\end{tabular}




\begin{tabular}{|c|c|c|c|c|}
\hline 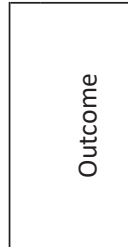 & 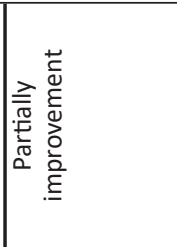 & 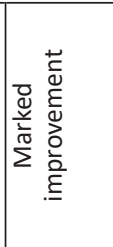 & 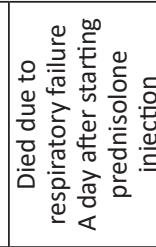 & 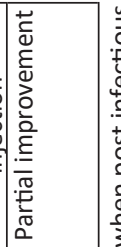 \\
\hline 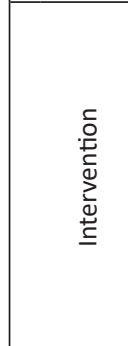 & 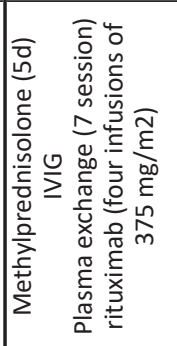 & 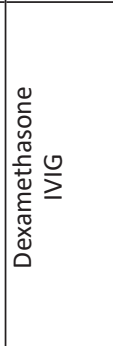 & 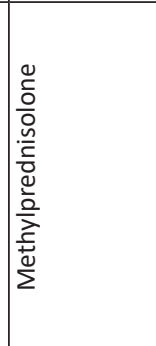 & 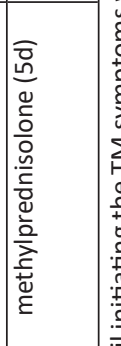 \\
\hline 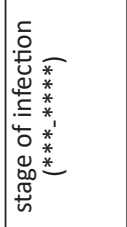 & 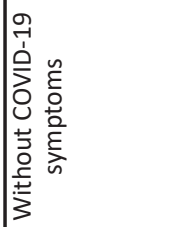 & 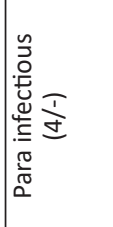 & 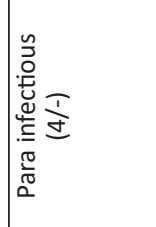 & 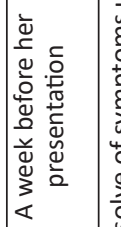 \\
\hline 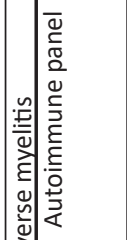 & 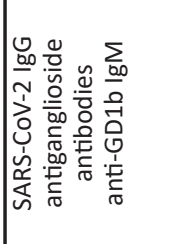 & 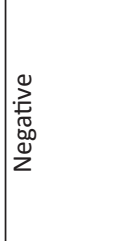 & 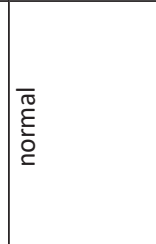 & 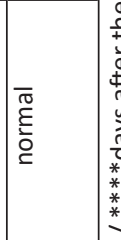 \\
\hline 递 & 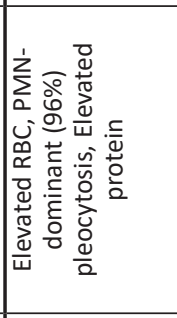 & \begin{tabular}{|l}
$\overline{\bar{\sigma}}$ \\
$\bar{\xi}$ \\
$\bar{z}$ \\
2
\end{tabular} & 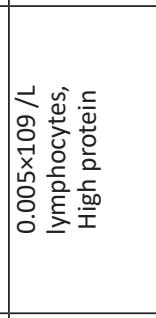 & 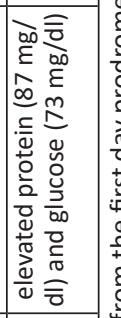 \\
\hline 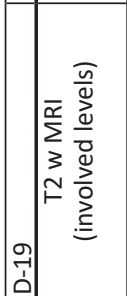 & 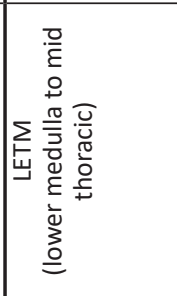 & 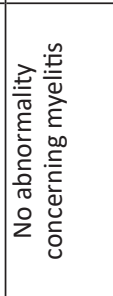 & $\sum_{1} \sum_{\hat{E}}$ & 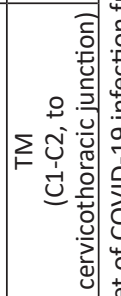 \\
\hline 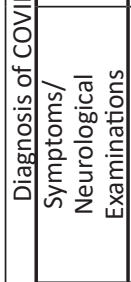 & 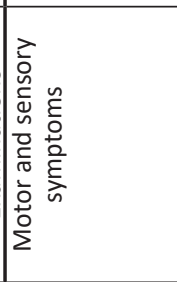 & 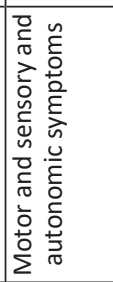 & 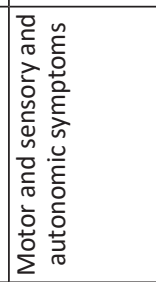 & 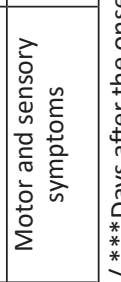 \\
\hline 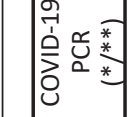 & \pm & $\mp$ & $I$ & $\mp$ \\
\hline 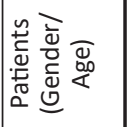 & 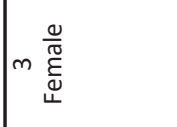 & in $\frac{0}{2 \pi}$ & م) & $\vec{b}$ \\
\hline 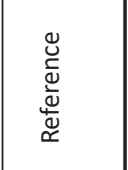 & 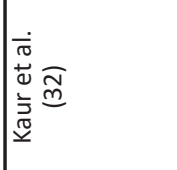 & 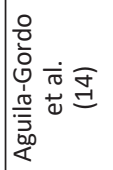 & 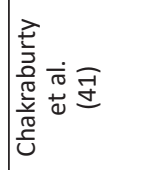 & 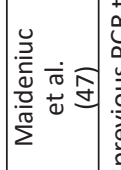 \\
\hline
\end{tabular}

be concerning because the age distribution is changing, and children might be at more risk of post/para COVID-19 TM (12).

Understanding TM's mechanism is necessary to face possible rising numbers of post/para COVID-19 infections and post COVID-19 vaccine TM.

Three suspicious mechanisms of nervous system involvement regarding COVID-19 infections are hematogenous route, olfactory route, and misdirected autoimmune response. Although each individual's real mechanism is not reliably distinguished, one or more of these mechanisms may be the underlying pathology (13).

In the context of COVID-19 induced encephalitis, a positive RT-PCR test for SARS-CoV-2 in CSF is highlighted by several authors, but it is not a common finding in the rest of COVID-19 neurological manifestations (14). Earlier available results support that COVID-19 induced encephalitis is mainly because of direct invasion of the virus, but other neurological manifestations are mainly post-infectious immunological reactions. Therefore, para/post-COVID-19 TM's diagnosis relies on the presence of COVID-19 CSF antibodies rather than RNA detection by CSF PCR (15). Nevertheless, according to a study, the highest positive rate of PCR is present when CSF samples are obtained five days after the onset of symptoms (16).

In the published literature, nine cases had positive throat COVID-19 swab PCR on their current neurological manifestation admission. Regarding available data, only one patient had positive SARSCoV-2 throat RT PCR a week after initiation of her symptoms (17), while almost all patients had positive COVID-19 throat RT-PCR during the first week of their COVID-19 related symptoms.

Previous studies showed that positive rate of nasopharyngeal RT-PCR assay is highest at days 0-7 (97.9\%), followed by $68.8 \%, 36.3 \%, 30.0 \%$, and $26.3 \%$ at days $8-14,15-21,22-28$, and $>28$, respectively. The median period between symptoms presence and positive SARS-CoV-2 RT-PCR results is 16 days, and the median period between symptoms presence and an effective negative SARS-CoV-2 RT-PCR result is 20 days. So negative throat RT-PCR tests do not rule out SARSCoV2 related cord inflammation (18).

Inflammation of the cord is defined by CSF pleocytosis or elevated immunoglobulin G ( $\mathrm{IgG}$ ) index. 
However, some patients presenting with TM may not fulfill all of the imaging and laboratory criteria. For example, a significant percentage of individuals with a clinical pattern that otherwise resembles TM does not meet the inflammatory features; therefore, the absence of inflammatory markers does not rule out TM, and further workup is required (9).

However, four patients had normal CSF pattern in our review; an oligoclonal band was present in two of them $(19,20)$. These results might support the theory of activated underlying autoimmune disease in these two patients.

The autoimmune panel was checked for 13 patients, which was negative for 10 cases $(76.92 \%)$. Lumbar puncture was not performed in one patient due to its contraindication (21).

Current published case reports support the hypothesis that post/para COVID-19 ATM results from the patient's body's immune reaction.

Three patients had positive serology findings: 1 case had lupus anticoagulant $\mathrm{Ab}(21)$, another case with antiganglioside $\mathrm{Ab} \&$ anti-GD1b IgM, and upbeat oligoclonal band in CSF (22) remaining case with MOG-IgG presence in serum (20).

Of course, literature support an increase of lupus anticoagulant and antinuclear antibodies in patients hospitalized with COVID-19 (23-25). A cohort study showed almost $87 \%$ of their ICU patients had positive lupus anticoagulants (26).

Zhou et al. (20) reported a 26 years old male presented with concomitant visual symptoms, including bilateral subacute sequential vision loss with right RAPD (relative afferent pupillary defect), retinal hemorrhage, and bilateral disc edema and venous congestion concomitant with progressive dry cough. CSF RT-PCR for SARS-CoV-2 was negative, but throat swab PCR for SARS$\mathrm{CoV}-2$ was positive on admission with the presence of respiratory symptoms a few days before his present admission. The authors considered the patient as a MOG-IgG-mediated disease due to clinical presentation and positive MOG antibody in serum (20). MOG-IgG antibodies located on the extracellular surface of myelin sheaths and oligodendrocytes target the MOG receptors. Its expression begins late in myelination, giving rise to the idea this protein can serve as a potential marker for oligodendrocyte maturation and may play a role in maintaining myelin sheaths (27). In the case above, two differential diagnoses are MOG antibody disease and neuromyelitis optica spectrum disorder (NMOSD). NMOSD is a rare syndrome that usually occurs in young patients, characterized by the combination of acute optic neuritis and transverse myelitis in demyelinating syndromes of the CNS. Myelin oligodendrocyte glycoprotein (MOG) is a potential target for demyelinating autoantibodies. To establish NMO following at list one of its clinical cores (optic neuritis, acute myelitis, area postrema, acute brain stem, and diencephalic clinical syndromes and symptomatic cerebral syndrome with NMSOD-typical brain lesions) concomitant with detecting AQP4-IgG in the serum and excluding alternative diagnosis is indicative (28). Despite this, having negative AQP4-IgG does not exclude NOM, and considering other clinical core characteristics is necessary $(28,29)$. Myelin oligodendrocyte glycoprotein antibody disorders (MOGAD) variants of demyelinating diseases usually involve a demonstration around the beginning of the third decade of life (30). A possible hypothesis for the abovementioned case is the activation of an underlying immunologic disease following COVID-19 infection. The hypothesis can justify a variety of responses to the COVID-19 infection in different patients.

Alketbi et al. (21) reported a 32 years old male with positive novel COVID-19 pharyngeal swab PCR on admission who developed acute transverse myelitis two days after initiation of respiratory symptoms. He had positive lupus anticoagulant and low protein $\mathrm{S}$ and tachypnea and tachycardia symptoms with high D-dimer levels and a positive pulmonary CT angiography suggestive of pulmonary emboli. The underlying coagulation disorder increases the suspicion that the etiology of symptoms may be regarding spinal infarction due to spinal venous thrombosis.

Another support for our hypothesis was that a seventy-year-old female developed acute motor axonal neuropathy (AMAN) variant of Guillain-Barré syndrome (GBS) fifteen days after initiation of COVID-19 related respiratory symptoms. Activation of anti-GD1 b IgM concomitant with positive COVID-19 IgM in plasma advocated a SARS-CoV-2-related acute AMAN/myelitis complex as a result of a post-infective autoimmune reaction mechanism (22).

Available evidence from three case reports discussed above might represent a possible connec- 
tion between the occurrence of ATM presentations and the presence of an underlying autoimmune condition that leads to infectious or para/post-infectious flare-up of underlying autoimmune disease by COVID-19 infection.

In the conclusion of published evidence, understanding the exact mechanism of COVID-19 induced ATM requires careful CSF study in viral RNA detection and immunoglobulins. Eventually, a definite diagnosis of the type of involvement associated with SARS-CoV-2 should be made by biopsy or autopsy (15).

Regarding many clues that had been discussed, the most probable etiology of TM in the setting of COVID-19 is immune-related mechanisms.

However, in the our case report the required evidence to relate transverse myelitis to the COVID-19 was limited; we started the treatment with methylprednisolone as guidelines are recommended. It seems the most effective treatment is beginning five days corticosteroid IV course as soon as possible and delayed initiation of corticosteroids is associated with unsatisfactory results $(15,22,31)$. even though a combination of IVIG with corticosteroid resulted in better outcomes (32), initial treatment with IVIG without corticosteroid did not have good results $(15,22,31)$.

Nevertheless, at acute transverse myelitis in adults, when sensory and motor function is completely lost, the methylprednisolone response is poor (33). Thus, in addition to high-dose glucocorticoid therapy, for patients who have acute TM with motor impairment or if symptoms are getting worsen, plasmapheresis or plasma exchange is recommended (34-36).

Many studies confirmed the good results of plasmapheresis or plasma exchange in patients with transverse myelitis (32,37-39). Unfortunate$1 y$, in our case, in the first session of plasmapheresis, the blood pressure decreased, and the plasmapheresis was interrupted. New lab data showed increases in creatinine.

We considered Rituximab to suppress COVID-19 induced cytokine release syndrome and treat post-COVID-19 immunological processes. Immune suppressions may be considered when symptoms are getting worsen (4). To date, the most frequently applied immunotherapies for neurological diseases are DNA-synthesis interferences like Azathioprine, Methotrexate, or immune cell deple- tions like Rituximab. Immune-based mediated theory of neurological manifestations of the COVID-19 can justify the effect of Rituximab in one case $(32,40)$. However, Kaur et al. (32) reported good Rituximab outcomes in one patient; our patient did not respond to it. One possibility is that Rituximab is effective when immune antibodies such as antiganglioside antibodies, anti-GD1b IgM are present in the serum. Although another cause delays in therapy and our patients were end-stage and developed multisystem organ failure when Rituximab began. Two days after initiation of the Rituximab, the patient complained of pleural pain and shortness of breathing. A chest CT scan confirmed left side pleural effusion. Rituximab was held immediately, and a surgical consult was requested.

The condition of our patient deteriorated rapidly. Unfortunately, the patient died due to multiple organ damage and respiratory failure. The outcome of patients in our review are summarized in Table 1. One patient died due to a relapse of her COVID infection (41). Seven patients did not have a complete improving trend. Out of them, one patient was discharged with unimprovement in his clinical findings (15), and six patients were discharged with partial improvement to some rate of clinical persistence in case of their ATM manifestations. Among these seven patients, five patients (71.42\%) had longitudinally extensive T2-hyper intensity ( $\geq 3$ or more vertebral segments) or multifocal lesions in spinal cord imaging and one case (14.28\%) with the presence of an oligoclonal band in CSF and positive antiganglioside $\mathrm{Ab} \&$ anti-GD1b IgM in serum (22).

The overall prognosis of idiopathic TM is better than what the initial symptoms might suggest, and our review confirms previous results. Nevertheless, when most of the level is involved, in about 40 percent of patients, some degree of disability will be persisted. Very rapid onset with complete paraplegia and spinal shock has been associated with poorer outcomes (42-44). Therefore, as our review demonstrated, longitudinally extensive and multifocal spinal cord lesions and CSF oligoclonal bands' presence were featured available in $71.42 \%$ of patients with para/post-COVID ATM influence disease severity, outcome, and recurrence. Nevertheless, long-term outcomes of post/para COVD19 TM will remain unknown. Further studies are 
needed to evaluate the long-term outcomes of these patients.

\section{CONCLUSIONS}

This literature review strongly support that post/para infectious COVID-19 transverse myelitis primary pathogenesis is immune-mediated.

We talked about a new probable mechanism that a silent underlying immune disease might impact patients' prognosis, and this concept may affect the strategic plan.

Concerning the global vaccination of COVID-19, we would be alert to face new challenges of post-vaccinal complications such as increasing immune-mediated diseases.

Overall outcomes of TM in the setting of TM are good. Nevertheless, it is vital to start the treat- ment as soon as possible, and immediate therapy is one of the most influential prognostic factors for outcomes. This case report bolds some concerns about aggressive immunotherapies might deteriorate subsisted COVID-19 pneumonitis.

Concerning all available data and tries to provide the appropriate and safe vaccine, more studies for the effect of aggressive immunotherapies on the treatment of complicated COVID-19 induced CNS complications are necessary. The cut point and indication for considering immunotherapy as treatment should be determined.

\section{Acknowledgement}

The authors acknowledge the victim's family who agreed to include their patient's information in the article.

Conflict of interest: none declared Financial support: none declared

\section{REFERENCES}

1. Durrani M, Kucharski K, Smith Z, Fien S. Acute Transverse Myelitis Secondary to Severe Acute Respiratory Syndrome Coronavirus 2 (SARS-CoV-2): A Case Report. Clin Pract Cases Emerg Med. 2020;4(3):344-8.

2. Aghagoli G, Gallo Marin B, Katchur NJ, Chaves-Sell F, Asaad WF, Murphy SA. Neurological Involvement in COVID-19 and Potential Mechanisms: A Review. Neurocrit Care. 2020:1-10.

3. Bhat A, Naguwa S, Cheema G, Gershwin ME. The epidemiology of transverse myelitis. Autoimmun Rev. 2010;9(5):A395-9.

4. West TW. Transverse myelitis - a review of the presentation, diagnosis, and initial management. Discov Med. 2013; 16(88):167-77.

5. Beh SC, Greenberg BM, Frohman T, Frohman EM. Transverse myelitis. Neurologic clinics. 2013;31(1):79-138.

6. Bakshi R, Kinkel PR, Mechtler LL, Bates VE, Lindsay BD, Esposito $\mathrm{SE}$, et al. Magnetic resonance imaging findings in 22 cases of myelitis: comparison between patients with and without multiple sclerosis. European Journal of Neurology. 1998;5(1):35-48.

7. Choi KH, Lee KS, Chung SO, Park JM, Kim YJ, Kim HS, et al. Idiopathic transverse myelitis: MR characteristics. AJNR. 1996;17(6):1151-60.

8. Greenberg BM, Frohman EM. Immune-mediated myelopathies. Continuum (Minneapolis). 2015;21(1 Spinal Cord Disorders):121-31.

9. de Seze J, Lanctin C, Lebrun C, Malikova I, Papeix C, Wiertlewski $\mathrm{S}$, et al. Idiopathic acute transverse myelitis: application of the recent diagnostic criteria. Neurology. 2005;65(12):1950-3.

10. Pidcock FS, Krishnan C, Crawford TO, Salorio CF, Trovato M, Kerr DA. Acute transverse myelitis in childhood: center-based analysis of 47 cases. Neurology. 2007;68(18):1474-80.

11. Boehmer TK, DeVies J, Caruso E, van Santen KL, Tang S, Black $\mathrm{CL}$, et al. Changing Age Distribution of the COVID-19 Pandemic - United States, May-August 2020. MMWR. 2020;69(39):1404-9.

12. Davies NG, Klepac P, Liu Y, Prem K, Jit M, Pearson CAB, et al. Age-dependent effects in the transmission and control of COVID-19 epidemics. Nature Medicine. 2020;26(8):1205-11.

13. Abdelaziz OS, Waffa Z. Neuropathogenic human coronaviruses: $A$ review. Reviews in Medical Virology. 2020;30(5).

14. Águila-Gordo D, Manuel Flores-Barragán J, Ferragut-Lloret F, et al. Acute myelitis and SARS-CoV-2 infection. A new etiology of myelitis? J Clin Neurosci. 2020;80:280-1.

15. Saberi A, Ghayeghran A, Hatamian H, Hosseini-Nejad M, Bakhshayesh Eghbali B. COVID-19-Associated Myelitis, Para/Post Infectious or Infectious Myelitis: A Case Report From the North of Iran. Caspian Journal of Neurological Sciences. 2020;6(2):132-8.

16. Kincaid O, Lipton HL. Viral myelitis: an update. Current Neurology and Neuroscience Reports. 2006;6(6):469.

17. Sotoca J, Rodríguez-Álvarez Y. COVID-19-associated acute necrotizing myelitis. Neurology $\otimes^{\circledR}$ Neuroimmunology \& Neuroinflammation. 2020;7(5).

18. Xiao AT, Tong YX, Gao C, Zhu L, Zhang YJ, Zhang S. Dynamic profile of RT-PCR findings from 301 COVID-19 patients in Wuhan, China: a descriptive study. Journal of Clinical Virology. 2020:104346.

19. Masuccio FG, Lo Re M, Bertolotto A, Capobianco M, Solaro C. Benign SARS-CoV-2 infection in MOG-antibodies associated disorder during tocilizumab treatment. Multiple Sclerosis and Related Disorders. 2020;46.

20. Zhou S, Jones-Lopez EC, Soneji DJ, Azevedo CJ, Patel VR. Myelin Oligodendrocyte Glycoprotein Antibody-Associated Optic Neuritis and Myelitis in COVID-19. J Neuroophthalmol. 2020;40(3):398-402.

21. AlKetbi R, AINuaimi D, AlMulla M, AlTalai N, Samir M, Kumar N, et al. Acute myelitis as a neurological complication of Covid-19: A case report and MRI findings. Radiol Case Rep. 2020;15(9):1591-5.

22. Masuccio FG, Barra M, Claudio G, Claudio S. A rare case of acute motor axonal neuropathy and myelitis related to SARS-CoV-2 infection. Journal of Neurology. 2020:1-4.

23. Bowles L, Platton S, Yartey N, Dave M, Lee K, Hart DP, et al. Lupus Anticoagulant and Abnormal Coagulation Tests in Patients with Covid-19. N Engl J Med. 2020 Jul 16;383(3):288-290.

24. Mantovani Cardoso E, Hundal J, Feterman D, Magaldi J. Concomitant new diagnosis of systemic lupus erythematosus and COVID-19 with possible antiphospholipid syndrome. Just a coincidence? A case report and review of intertwining pathophysiology. Clinical Rheumatology. 2020;39(9):2811-5.

25. Gazzaruso C, Mariani G, Ravetto C, Malinverni L, Tondelli E, Cerrone $\mathrm{M}$, et al. Lupus anticoagulant and mortality in patients hospitalized for COVID-19. Journal of Thrombosis and Thrombolysis. 2020.

26. Helms J, Tacquard C, Severac F, Leonard-Lorant I, Ohana M, Delabranche $X$, et al. High risk of thrombosis in patients with severe SARS-CoV-2 infection: a multicenter prospective cohort study. Intensive Care Medicine. 2020;46(6):1089-98. 
27. Narayan R, Simpson A, Fritsche K, Salama S, Pardo S, Mealy M, et al. MOG antibody disease: A review of MOG antibody seropositive neuromyelitis optica spectrum disorder. Multiple sclerosis and Related Disorders. 2018;25:66-72.

28. Haase CG, Schmidt S. Detection of brain-specific autoantibodies to myelin oligodendrocyte glycoprotein, S100beta and myelin basic protein in patients with Devic's neuromyelitis optica. Neuroscience Letters. 2001;307(2):131-3.

29. Wingerchuk DM, Banwell B, Bennett JL, Cabre P, Carroll W, Chitnis $\mathrm{T}$, et al. International consensus diagnostic criteria for neuromyelitis optica spectrum disorders. Neurology. 2015; 85(2):177-89.

30. Reindl M, Waters P. Myelin oligodendrocyte glycoprotein antibodies in neurological disease. Nature Reviews Neurology. 2019; 15(2):89-102.

31. Zachariadis A, Tulbu A, Strambo D, Dumoulin A, Di Virgilio G. Transverse myelitis related to COVID-19 infection. Journal of Neurology. 2020;267(12):3459-61.

32. Kaur H, Mason JA, Bajracharya M, McGee J, Gunderson MD, Hart $\mathrm{BL}$, et al. Transverse myelitis in a child with COVID-19. Pediatric Neurology. 2020.

33. Greenberg BM, Thomas KP, Krishnan C, Kaplin Al, Calabresi PA, Kerr DA. Idiopathic transverse myelitis: corticosteroids, plasma exchange, or cyclophosphamide. Neurology. 2007;68(19):1614-7.

34. Bigi S, Banwell B, Yeh EA. Outcomes after early administration of plasma exchange in pediatric central nervous system inflammatory demyelination. Journal of Child Neurology. 2015;30(7):874-80.

35. Greenberg BM. Treatment of acute transverse myelitis and its early complications. Continuum (Minneapolis). 2011;17(4):733-43.

36. Gwathmey K, Balogun RA, Burns T. Neurologic indications for therapeutic plasma exchange: an update. Journal of Clinical Apheresis. 2011;26(5):261-8.

37. Sarma D, Bilello LA. A Case Report of Acute Transverse Myelitis Following Novel Coronavirus Infection. Clin Pract Cases Emerg Med. 2020;4(3):321-3.
38. Baghbanian SM, Namazi F. Post COVID-19 longitudinally extensive transverse myelitis (LETM) - a case report. Acta Neurologica Belgica. 2020.

39. Valiuddin H, Skwirsk B, Paz-Arabo P. Acute transverse myelitis associated with SARS-CoV-2: A Case-Report. Brain Behav Immun Health. 2020;5:100091.

40. Berlit P, Bösel J, Gahn G, Isenmann S, Meuth SG, Nolte CH, et al. Neurological manifestations of COVID-19 - guideline of the German Society of Neurology. Neurological Research and Practice. 2020;2:51.

41. Chakraborty U, Chandra A, Ray AK, Biswas P. COVID-19associated acute transverse myelitis: a rare entity. BMJ Case Reports CP. 2020;13(8):e238668.

42. Defresne P, Hollenberg H, Husson B, Tabarki B, Landrieu P, Huault $\mathrm{G}$, et al. Acute transverse myelitis in children: clinical course and prognostic factors. Journal of Child Neurology. 2003;18(6):401-6.

43. Ganelin-Cohen E, Konen O, Nevo Y, Cohen R, Halevy A, Shuper A, et al. Prognostic Parameters of Acute Transverse Myelitis in Children. Journal of Child Neurology. 2020;35(14):999-1003.

44. Kaplin Al, Krishnan C, Deshpande DM, Pardo CA, Kerr DA. Diagnosis and management of acute myelopathies. The Neurologist. 2005;11(1):2-18.

45. Munz M, Wessendorf S, Koretsis G, Tewald F, Baegi R, Krämer S, et al. Acute transverse myelitis after COVID-19 pneumonia. J Neurol. 2020;267(8):2196-7.

46. Chow CCN, Magnussen J, Ip J, Su Y. Acute transverse myelitis in COVID-19 infection. BMJ Case Rep. 2020;13(8).

47. Maideniuc $C$, Memon $A B$. Acute necrotizing myelitis and acute motor axonal neuropathy in a COVID-19 patient. Journal of Neurology. 2020:1-3. 